\title{
LOS DEPARTAMENTOS DE ORIENTACIÓN EN LOS IES: ESTUDIO DE LAS DISOCIACIONES ENTRE PERCEPCIONES REALES Y NORMATIVA LEGAL
}

\author{
Rufino Cano González \\ Universidad de Valladolid
}

\begin{abstract}
RESUMEN. El hecho de que estemos a punto de cumplir los diez primeros años de implantación de la LOGSE, es motivo más que suficiente para reflexionar, desde la teoría, y evaluar, desde la práctica, su mutua corresponsabilidad en uno de los ámbitos de la estructura organizativa de los IES especialmente cuidado, como es el caso de los Departamentos de Orientación. Nuestro objetivo, pues, es evaluar la posible conjunción o disociación del binomio, teoría-práctica, mediante un análisis comparativo entre lo prescrito en la normativa legal promulgada por la Administración Pública y lo descrito y percibido a través de las experiencias de Orientación que se vienen desarrollando en los Institutos de Enseñanza Secundaria de Valladolid y provincia, con la intención última de formular propuestas y recomendaciones de mejora basadas en los resultados del estudio que hemos realizado.

ABSTRACT. The fact that we're just to celebrate the first ten years of the LOGSE implementation, is reason enough to reflect, from the theory, and evaluate, from the practice, the mutual responsibility in one of the major levels of the organisational structure of the IES (Institutes of Secondary Education), that is the case of the Orientation Department. Our aim is, then, to evaluate this relationship, theory-practice, through a comparative analysis between the prescriptive legal normative from the Public Administration and what has been described in the different experiences in Orientation developed in the institutes of Secondary Education in Valladolid and its province, with the ultimate intention of formulating proposals and improvement recommendations basalt on the results of our research.
\end{abstract}

\section{Introducción}

Desde la aparición de la Orden Ministerial de 4 de junio de 1987, por la que se convocan Proyectos de Orientación Educativa en Centros de Enseñanza Media (B.O.E., 66-87), han pasado 13 años. Durante este breve pero intenso periodo se han producido importantes cambios en nuestro Sistema Educativo como consecuencia de la implementación gradual de una nueva Ley Educativa, la LOGSE, a través de la cual se pretende no solamente ordenar y estructurar, de forma general, el Sistema Educativo, sino, también, y primordialmente, mejorar la calidad y eficiencia de la enseñanza y el desarrollo pleno de la actividad educativa desde un planteamiento comprensivo y diversifi- 
cador más en consonancia con los tiempos actuales. Y es que la educación y, por tanto, la orientación ,se han de proyectar hacia los jóvenes como la verdadera llave para abrir nuevos horizontes de perfección y de posibilidades para su desarrollo y formación personal, profesional y social, desde los cuales, juntamente con sus padres, profesores y tutores, tendrán que tomar, conscientemente, decisiones de gran trascendencia para su futuro a la hora de elegir opciones formativas y profesionales coherentes.

Junto a un conjunto de principios fundamentales (personalización de la enseñanza, autonomía pedagógica de los Centros, innovación e investigación educativa, orientación educativa y profesional e intervención psicopedagógica), en los que se asienta la Reforma y se fundamenta la actividad educativa, adquieren carta de atención preferente una serie de factores que favorecen la calidad de la oferta educativa en el conjunto de los niveles de la enseñanza obligatoria (Educación Primaria y Educación Secundaria Obligatoria), y no obligatoria (Bachillerato en cualquiera de sus modalidades y Formación Profesional Específica: Ciclos Formativos de Grado Medio). Estos factores de apoyo y asesoramiento se proyectan, de manera especial, en torno a siete grandes proposiciones o líneas básicas de actuación (LOGSE, art. 55), de entre las que nos interesa destacar, en este momento, aquella que se refiere, genéricamente, a la Orientación Psicopedagógica, en el doble ámbito de la orientación educativa y de la intervención psicopedagógica.

En este nuevo marco ofertado por la Reforma del Sistema Educativo y enriquecido gradual y sistemáticamente por sucesivas aportaciones de interés relevante (Libro Blanco para la Reforma del Sistema Educativo. MEC, 1989; La Orientación Educativa y la Intervención Psicopedagógica. MEC, 1990; Orientación y Tutoría. MEC, 1992; Ley Orgánica 9/1995 de 20 de noviembre, de la Participación, Evaluación y el Gobierno de los Centros Docentes; Real Decreto 83/1996, de 26 de enero, por el que se aprueba el Reglamento Orgánico de los Institutos de Educación Secundaria; Resolución de 29 de abril de 1996, de la Dirección General de Centros Escolares, sobre organización de los Departamentos de Orientación de los Institutos de Educación Secundaria, y Circular de 30 de abril de 1996, de la Dirección General de Renovación Pedagógica, por la que se dictan instrucciones sobre el Plan de actividades de los Departamentos de Orientación de los Institutos de Educación Secundaria, etc.), aparece el Departamento de Orientación (en adelante, D.O.), "como un recurso necesario, como una estructura apta para el desarrollo de amplias y complejas funciones, como uno de los requerimientos de carácter educativo cuya implementación, mejora y consolidación ha de confluir en una propuesta de enseñanza y educación cualitativamente mejor" (CANO, R. y BORONAT, J. 1998: 168). Y todo ello, porque cada vez somos más conscientes del valor que la Orientación Psicopedagógica tiene en el desarrollo del derecho a la educación que asiste a todos los alumnos: "A recibir orientación escolar y profesional para conseguir el máximo desarrollo personal, social y profesional según sus capacidades, aspiraciones e intereses..." (Art. 14 del R.D. 732/1995, de 5 de mayo, por el que se establecen los derechos y deberes de los alumnos y las normas de convivencia en los Centros.).

Muchos son los autores que, desde el campo conceptual y/o de la investigación aplicada, se han acercado a los D.O. para calar en su esencia y responder mejor a una serie de demandas inducidas por las preguntas lashwellianas de todos ya suficientemente 
conocidas: ¿Qué concepto de Orientación Psicopedagógica tienen el MEC ., las CC.AA y los profesionales de la educación? ¿Quién/es realizan específicamente esta tarea? ¿Cómo adquiere presencia real en los Centros? ¿Cuándo hay que comenzar? ¿Dónde están localizados sus escenarios de intervención? ¿Por qué tenemos que orientar?

La noción, idea o tesis que la comunidad científica tiene acerca de los D.O., en tanto que puntales destacados en la organización de los Centros de Enseñanza Secundaria, es que estos se configuran y se consolidan como "el espacio institucional desde donde se articulan las funciones de orientación y tutoría, así como también las de una oferta curricular adaptada y diversificada" (Orientación y Tutoría. MEC, 1992:35). Dichas funciones se han de incardinar dentro de la Programación General Anual (PGA) y han de estar incluidas en los niveles programático-curriculares de Centro, de Etapa (organización y coordinación de la orientación psicopedagógica; plan de acción tutorial; atención a la diversidad: necesidades educativas especiales y diversificación curricular; agrupamientos de alumnos; organización de espacios; programación de actividades complementarias; programación de actividades extraescolares; programaciones didácticas de los departamentos) y de Area, para darle una operatividad y una funcionalidad propiamente educativas.

En este sentido, no se puede entender ni justificar la intervención psicopedagógica aislada de la práctica educativa que se genera en los Centros y, más concretamente, de la práctica educativa del profesor con el grupo-clase de forma colectiva $o$, de manera individual, con cada uno de los alumnos. No es correcto y, por tanto, nada recomendable que el Orientador se comprometa y asuma de forma individual una serie de tareas con los alumnos sin tener en cuenta las preocupaciones, necesidades y funciones del profesorado responsable, por cuanto que la Orientación se entiende como algo conjunto y complementario, como un apoyo integrado de colaboración con el profesorado, las familias y los propios alumnos, que adquiere su pleno sentido en el "continuum" de la actividad educativa.

La LOGSE y la literatura educativa que se ha ido gestando, inicialmente de forma prescriptiva y con cierta fluidez legisladora a través de la normativa oficial, y, sucesivamente, mediante los distintos foros de debate y compendios bibliográficos al uso, defienden con claridad meridiana el rol de los Orientadores y su razón de ser en cuanto que hacen más competente al profesorado en la tarea de individualización de la enseñanza (atención a la diversidad), pudiéndose identificar el concepto de Orientación con el mismo proceso de enseñanza-aprendizaje que se realiza en los Centros. Así, podemos afirmar con absoluta rotundidad que la Orientación es un asunto de Centro que compete a toda la comunidad educativa, incluso a sus Órganos de Gobierno (Unipersonales y Colegiados) y de Coordinación Docente (Departamentos Didácticos, Comisión de Coordinación Pedagógica, Junta de Profesores y Equipos de Tutores).

En consecuencia, toda la institución, todo el profesorado, se han de "sentir" y "ser" orientadores para cuestionarse su trabajo (sobre todo, el qué y el cómo) y tomar aquellas decisiones de mejora que les permita perfeccionar su propia práctica educativa. Es de este estado de incertidumbre, que necesariamente se tiene que producir, de donde debe surgir la reflexión, la investigación, el perfeccionamiento y la innovación; "no desde el dogmatismo, desde la seguridad, desde la cerrazón", como afirma SANTOS GUERRA. 
En definitiva, los D.O. de los Centros de Secundaria, en cuanto que órganos de planificación y coordinación de la acción orientadora, de la función tutorial y de la oferta curricular adaptada y diversificada, "se constituyen en uno de los instrumentos más idóneos de apoyo y dinamización de las instituciones educativas no universitarias y en uno de los resortes más favorecedores de la calidad y mejora de la enseñanza y oferta educativa", CANO GONZÁLEZ y BORONAT MUNDINA (1998: 173). Su presencia e intervención se justifica no al margen, sino en plena integración y simbiosis dentro del propio Proyecto Educativo y de la actividad docente.

El Departamento de Orientación se erige, así, en el núcleo básico o eje transversal de la organización pedagógica del Centro. Desde él, y atendiendo las demandas y necesidades de los distintos sectores educativos "intra Centro", se genera, se desarrolla y se proyecta la acción orientadora y de apoyo, siempre deseable desde una triple vertiente: preventiva o anticipadora, curativa o terapéutica y de desarrollo personal o integradora.

\section{Planteamiento del estudio}

La multiplicidad y diversidad de tareas o funciones técnicas y de apoyo, con una formulación demasiado compleja y ambiciosa, que la Reforma Educativa vigente ha asignado a los D.O. de los IES, es decir, al conjunto de sus componentes y no solamente al Orientador, como a veces erróneamente se pretende deducir, en relación con el Centro, Equipo Directivo, Departamentos Didácticos, Comisión Técnica de Coordinación Pedagógica, profesores, alumnos y familia, puede distorsionar la labor de estos órganos de planificación orientadora y de apoyo y asesoramiento educativo, cargando excesivamente sus esfuerzos en favor de unas áreas de atención psicopedagógica ciertamente importantes, en detrimento de aquellas otras, tal vez a primera vista menos exigentes pero, a veces, primordiales para el desarrollo de una labor educativa mucho más eficaz y pedagógicamente más valiosa y oportuna.

Si esto se produce, teóricamente, a nivel de propuesta legislativa y documental (ya veremos luego hasta qué punto), nos interesa conocer qué está sucediendo en el ámbito de la acción aplicada y, consecuentemente, plantearnos, en este sentido, algunas preguntas, como por ejemplo: ¿cuál es la realidad de la intervención orientadora en el conjunto de los IES? (nosotros, a modo de ejemplo, nos referiremos a los ubicados en Valladolid y su provincia). ¿Qué tipo de tareas y programas se están Ilevando a cabo en este momento? ¿En cuáles se está invirtiendo mayor esfuerzo, y por qué? ¿Qué campos de atención psicopedagógica apenas tienen resonancia? ¿Qué se debería hacer si en la práctica existiera una descompensación considerable entre la atención a unas y otras funciones? ¿Cuál es el compromiso real del profesorado con respecto a los D.O.? ¿y el de los Equipos Directivos? ¿Qué habría que modificar y cuál potenciar? ¿Qué modelo de Departamento subyace?. Son, ciertamente, cuestiones interesantes que acaparan nuestra atención y que, algunas de ellas, intentaremos dilucidar en este artículo.

\section{Objetivos}

Desde estas concepciones con referencia a los D.O., y teniendo en cuenta que el estudio que presentamos constituye, simplemente, una aproximación o acercamiento a 
dos mundos deseablemente dependientes y necesariamente inter e intra relacionados, -"la teoría orientadora como posibilidad y la práctica psicopedagógica como realidad" $^{\prime \prime}$, nuestra intención no es otra que colaborar en los trabajos e investigaciones en curso y aportar nuestro grano de arena en esta temática. En este sentido, hemos planteado la investigación sobre cuatro puntos u objetivos muy específicos y concretos:

1 . Percatarse del conjunto de las funciones que la LOGSE y la normativa legal que se ha ido generando en estos últimos años asignan a los D.O. de los IES (teoría orientadora como posibilidad. Ámbito normativo-teórico).

$2^{\circ}$. Conocer la labor que están desarrollando, a nivel de experiencias concretas, los D.O. de los IES de Valladolid y provincia (teoría psicopedagógica como realidad. Ámbito institucional-descriptivo), y su adscripción y representatividad en los ocho ámbitos temáticos que se citan (con especial atención a los tres primeros), analizando y valorando las acciones que se proyectan en cada uno de ellos: 1. Plan de Acción Tutorial. 2. Proceso de Enseñanza-aprendizaje. 3. Plan de Orientación Académico-profesional. 4. Atención a la Diversidad. 5. Investigación e Innovación Educativa. 6. Evaluación Psicopedagógica. 7. Formación permanente del Profesorado del Centro. 8. Apoyo y Orientación a las familias.

$3^{\circ}$. Reflexionar, críticamente, sobre el marco institucional normativo (documentos legales) y práctico descriptivo (lo que se está haciendo en los Centros), con la intención de contribuir a la mejora de la Orientación en sus facetas organizativa y psicopedagógica.

$4^{\circ}$. A la vista de los resultados obtenidos, y teniendo presentes las opiniones de los Orientadores, formular propuestas y recomendaciones de mejora para futuras intervenciones en el área de la Orientación Psicopedagógica o, en su caso, servir de sugerencias y ayuda a otros profesionales de zonas, comarcas y comunidades autónomas diferentes.

\section{Procedimiento metodológico}

El presente trabajo se ubica, inicialmente, en un campo de investigación mucho más amplio que el que aquí presentamos. Forma parte de una investigación que afecta, globalmente, al proceso de implantación y desarrollo de la Orientación Educativa e Intervención Psicopedagógica en contextos escolares no universitarios. Se trata, pues, de un estudio enmarcado en un horizonte más complejo y diverso pero, a la vez, íntimamente relacionado y enriquecedor.

Hecha esta pequeña pero necesaria anotación, hemos de decir, refiriéndonos a la metodología propia de este trabajo, que un primer paso corresponde al diseño, elaboración y aplicación de un instrumento básico de investigación, apto para penetrar y profundizar en el tema que nos proponemos, a través de soportes objetivos de información directa y actualizada. En consecuencia, y siguiendo la metodología de estudios descriptivos basados en encuestas, hemos recurrido a la confección de un cuestionario para la recogida de datos (como sustituto de la entrevista oral que no hemos podido realizar por razones de tiempo) que nos va a permitir vaciar, con cierta agilidad, su aporte de contenido en un molde fácilmente interpretable. 
El formato de evaluación (cuestionario) se divide en seis grandes apartados. Aquí, solamente, enunciaremos sus epígrafes generales sin entrar a especificar los descriptores propios de cada uno de ellos: 1. Datos referidos al Centro. 2. Datos referidos al Orientador. 3. Repertorio indicativo de las funciones y tareas propias del Departamento de Orientación (indicando en qué consisten, a quiénes se dirigen y cómo se suelen desarrollar). 4. Datos específicos (ya enunciados en el objetivo $n^{\circ} 2$ y que hacen referencia a la labor que están desarrollando los D.O. de los IES de Valladolid y provincia con referencia clara a los ocho ámbitos de intervención que ya hemos comentado más arriba). 5. Tareas y funciones externas al Centro en las que ha estado o está actualmente implicado el Departamento de Orientación. 6. Síntesis (tres preguntas abiertas en las que el Orientador ha de concretar su posicionamiento sobre una serie de cuestiones que afectan a la acción tutorial y a la orientación educativa).

La población de referencia, y que en este caso nos sirve de muestra, comprende a los Orientadores que actualmente están al frente de los Departamentos de Orientación de los IES de Valladolid y provincia, ubicados en núcleos o zonas de asentamiento urbanos, semiurbanos y rurales; en total 31 Orientadores (20 en la ciudad y 11 en la provincia) para los 31 Institutos. En todos se presta apoyo a la integración y en algunos este apoyo adquiere un carácter más selectivo al dirigirse a alumnos con deficiencias o minusvalías muy específicas, fundamentalmente visuales y auditivas.

Un segundo paso corresponde al análisis de contenido del cuestionario, a la evaluación de los resultados obtenidos y a las propuestas de mejora, armonización y optimización de las tareas del Orientador y áreas de intervención, si hubiere lugar.

A continuación, y después de habernos referido, brevemente, a aspectos e indicadores propiamente metodológicos, pasamos a conocer la labor que están desarrollando, a nivel de experiencias concretas, los D.O. de los IES de Valladolid y provincia y su adscripción y representatividad en los ocho campos de referencia ya descritos. Para ello, y en aras a facilitar las cosas, nos serviremos de una sencilla tabla que hemos confeccionado, en la que se registran los planes de acción, concretos y reales, que, en estos momentos, se están desarrollando, con distinta incidencia o resonancia como es, por otra parte, lógico que así sea.

\section{Resultados obtenidos en la investigación}

Seguidamente, iremos asignando, a cada uno de los cuatro objetivos que hemos planteado, las respuestas de que nos hemos hecho eco en el trabajo que hemos desarrollado, y su interpretación desde el análisis comparativo ya indicado más arriba, permaneciendo al margen de esta tarea el primero de los objetivos por cuanto que su enunciado se refiere, exclusivamente, a la vertiente normativo-teórica y no a la acción práctica real. No obstante, haremos una clara referencia aludiendo al contenido normativo del mismo como proposición comparativa para posteriores análisis y valoraciones. Para evitar ser reiterativos en la redacción de los objetivos, enunciaremos, a modo de recuerdo, cada uno de ellos con sus primeras palabras introductorias, siguiendo el mismo orden que hemos utilizado en su presentación preliminar: 
5.1. Objetivo $n^{\circ}$ 1: "Percatarse del conjunto de las funciones que la LOGSE y la normativa legal..."

¿Qué nos adelanta la norma? Desde el punto de vista de los D.O., la Orientación Psicopedagógica (orientación educativa e intervención psicopedagógica) se explicita, clara y determinantemente, desde tres ámbitos de actuación (amén de otros a los que posteriormente nos hemos de referir) con sus respectivas funciones. Veamos, a continuación, con detalle, el plan de actividades propuesto por el MEC para los D.O. de los IES, inicialmente recogido en el cuadro que unas líneas más abajo adjuntamos y, posteriormente, complementado por los dos siguientes que comentaremos un poco más adelante.

Con respecto al apoyo que ha de prestar el D.O. al Plan de Orientación Académica y Profesional, (primer ámbito de actuación), es necesario recordar que la Orientación en este campo debe entenderse como un proceso que ha de extenderse y desarrollarse durante toda la enseñanza secundaria, adquiriendo un matiz especialmente relevante en los momentos en los que los alumnos deben elegir materias optativas o en aquellos otros en los que la elección entre distintas opciones puede condicionar, en gran medida, el futuro académico y profesional de los estudiantes: itinerarios académicos en Bachillerato o Ciclos Formativos de Formación Profesional. Su finalidad es que ha de contribuir a facilitar la "toma de decisiones" de cada alumno respecto a su itinerario académico y profesional, a través de una serie de actividades y actuaciones, especificando las líneas prioritarias para cada etapa, ciclo y curso. Dicha "toma de decisiones", consiste en una propuesta colegiada de la Junta de Profesores, en la que, teniendo en cuenta las expectativas manifestadas por el propio alumno, se le recomendarán las opciones educativas o profesionales más acordes con sus capacidades, intereses y posibilidades.

\section{Cuadro no 1}

\section{AMBITOS DE ACTUACION Y FUNCIONES DE INTERVENCION DEL DEPARTAMENTO DE ORIENTACION DE LOS I.E.S.}

\begin{tabular}{|c|c|}
\hline AMBITO № 1 & FUNCIONES \\
\hline $\begin{array}{l}\text { PLAN DE ORIENTACION } \\
\text { ACADEMICA Y } \\
\text { PROFESIONAL }\end{array}$ & $\begin{array}{l}\text { * Elaboración del POAP (Plan de Orientación Académica y } \\
\text { Profesional), de acuerdo con los criterios establecidos por el } \\
\text { Claustro, las aportaciones de los Tutores y las directrices ema- } \\
\text { nadas de la CCP (Comisión de Coordinación Pedagógica). } \\
\text { * Inclusión del POAP en el PCE (Proyecto Curricular de Etapa). } \\
\text { * Información sobre el mundo laboral y salidas profesionales. } \\
\text { * Participación en la elaboración del Consejo Orientador al } \\
\text { finalizar la Enseñanza Secundaria Obligatoria y al término de } \\
\text { los Programas de Garantía Social. } \\
\text { * Participación en la evaluación del POAP y elaboración de la } \\
\text { Memoria final (síntesis reflexiva sobre: logros alcanzados, difi- } \\
\text { cultades encontradas, factores influyentes en ambos casos, } \\
\text { aspectos a modificar, etc.). }\end{array}$ \\
\hline
\end{tabular}


Otro de los ámbitos de actuación fijado por la norma, y que afecta directamente a los D.O., es el que se refiere al Apoyo al Proceso de Enseñanza-Aprendizaje. Una de las actividades propias de estos departamentos, tal vez de la más relevantes por su especificidad, exigencia técnica y profesional en su aplicación y valoración, sea la que se refiere a la "Evaluación Psicopedagógica" (prescriptiva para la incorporación de los alumnos a un programa de Diversificación Curricular, de Garantía Social o para poner en marcha un proceso de Adaptación Curricular individualizada), como determinante de la posterior adopción de medidas educativas específicas con alcance a distintas áreas del currículo para aquellos alumnos y alumnas que evidencien problemas serios en el progreso y desarrollo normal de sus aprendizajes.

Amén de este tipo de intervención psicopedagógica, de rasgo eminentemente terapéutico, existen otros modelos, como se podrá observar en el cuadro no2, mucho más en la línea preventiva (prevención y detección temprana de posibles problemas) y de asesoramiento al profesorado para que con la ayuda necesaria puedan responder a las dificultades de aprendizaje que presenten los alumnos desde la perspectiva del currículo ordinario.

\section{Cuadro no 2}

\begin{tabular}{|c|c|}
\hline \multicolumn{2}{|c|}{$\begin{array}{c}\text { ÁMBITOS DE ACTUACIÓN Y FUNCIONES DE INTERVENCIÓN DEL } \\
\text { DEPARTAMENTO DE ORIENTACIÓN DE LOS I.E.S. }\end{array}$} \\
\hline ÁMBITO № 2 & FUNCIONES \\
\hline $\begin{array}{l}\text { APOYO AL } \\
\text { PROCESO DE } \\
\text { ENSEÑANZA- } \\
\text { APRENDIZAJE }\end{array}$ & $\begin{array}{l}\text { * Medidas preventivas de apoyo: } \\
\text { - Prevención y detección temprana de problemas de aprendizaje. } \\
\text { - Información y asesoramiento al profesorado sobre: identificación } \\
\text { de capacidades presentes en los objetivos generales que se deben } \\
\text { destacar, secuencia de contenidos y objetivos, formas organizati- } \\
\text { vas, orientaciones metodológicas, criterios y procedimientos de } \\
\text { evaluación y seguimiento. } \\
\text { * Medidas de apoyo ordinario: } \\
\text { - Asesoramiento al profesorado para que puedan responder a las } \\
\text { dificultades de aprendizaje que presenten los alumnos desde la } \\
\text { perspectiva del currículo ordinario. } \\
\text { - Organización y diseño de actividades de recuperación, refuerzo y } \\
\text { ampliación. } \\
\text { - Colaboración en los niveles de planificación del Centro: Proyec- } \\
\text { to Educativo, Proyectos Curriculares de Etapa, Programación de } \\
\text { la actividad docente en el aula. } \\
\text { * Medidas de apoyo extraordinario para los alumnos que se } \\
\text { encuentren en alguna de estas situaciones: } \\
\text { - Que permanezcan un año más en un Ciclo o curso. } \\
\text { - Que requieran adaptaciones curriculares. } \\
\text { - Que accedan a un programa de Diversificación Curricular. } \\
\text { * Medidas generales y específicas de Atención a la Diversidad } \\
\text { social y cultural: } \\
\text { - Apoyo al profesorado } \\
\text { - Trabajo directo con los alumnos. } \\
\text { * Evaluación Psicopedagógica en colaboración con el profesorado. }\end{array}$ \\
\hline
\end{tabular}


Por último, el Plan de Acción Tutorial. Es el marco en el que se especifican los criterios de la organización y las líneas prioritarias de funcionamiento de la tutoría en el Centro. Su contenido incluye una serie de actuaciones que aseguran la coherencia educativa en el desarrollo de las programaciones y la práctica docente del aula por parte del profesorado del grupo, especialmente en lo relacionado con los contenidos y objetivos didácticos, procesos de evaluación, aspectos metodológicos, organizativos y de materiales didácticos, incorporación de los temas transversales, oferta de materias optativas, actividades en relación con la orientación académica y profesional, actividades en relación con el plan de acción tutorial, actuaciones para atender individualmente a los alumnos, etc.

\section{Cuadro no 3}

\begin{tabular}{|l|l|}
\hline \multicolumn{2}{|c|}{$\begin{array}{c}\text { ÁMBITOS DE ACTUACIÓN Y FUNCIONES DE INTERVENCIÓN DEL } \\
\text { DEPARTAMENTO DE ORIENTACIÓN DE LOS I.E.S. }\end{array}$} \\
\hline \multicolumn{1}{|c|}{ ÁMBITO NNo 3 } & \multicolumn{1}{c|}{ FUNCIONES } \\
\hline & * Promover e impulsar la programación de la acción tutorial \\
& $*$ Elaboración de propuestas al PAT (Plan de Acción Tutorial) con \\
& aportaciones de los Tutores. \\
& * Elaboración, desarrollo y evaluación del PAT, de acuerdo con las \\
& directrices de la CCP (Comisión de Coordinación Pedagógica). \\
PLAN DE & Incorporación a la propuesta de Proyecto Curricular que se pre- \\
ACCIÓN & sente al Claustro para su aprobación. \\
TUTORIAL & Facilitar a los Tutores las técnicas e instrumentos necesarios para \\
& su desarrollo. \\
& Facilitar, fomentar y cooperar en las relaciones Tutor-Familia. \\
& * Colaboración con el Jefe de Estudios como coordinador del Plan \\
& de Acción Tutorial. \\
& * Elaboración de un Memoria final de curso (síntesis reflexiva sobre: \\
& logros alcanzados, dificultades encontradas, factores influyentes \\
& en ambos casos, aspectos a modificar, etc.). \\
\hline
\end{tabular}

Fuente: R.D. 929/1993 de 18 de junio. Reglamento Orgánico de los IES de 1996. Circular de 30 de abril de 1996.

Conviene recordar que para cada uno de estos tres ámbitos (de acuerdo con la Circular de 30 de abril de 1996, de la Dirección General de Renovación Pedagógica, sobre la elaboración y desarrollo del Plan de Actividades de los Departamentos de Orientación, BOMEC, 13-5-96), se han de especificar, en dicho Plan, cuatro aspectos fundamentales: $1^{\circ}$. Objetivos que se pretenden conseguir. $2^{\circ}$. Actuaciones que se van a desarrollar, señalando todo tipo de colaboraciones necesarias (Equipo Directivo, Consejo Escolar, Comisión de Coordinación Pedagógica, Departamentos Didácticos, Departamento de Actividades Complementarias y Extraescolares, Tutores, Juntas de Profesores) y especificando, con claridad, cuáles de éstas son competencia del propio D.O., de cada componente del mismo y/o de otros profesores. $3^{\circ}$. Procedimientos para llevar a cabo su seguimiento y evaluación. 4º. Temporalización. 
5.2. Objetivo $n^{\circ}$ 2: “Conocer la labor que están desarrollando, a nivel de experiencia concretas, los Departamentos de Orientación..."

Abordaremos este segundo objetivo partiendo de unas sencillas preguntas: ¿se ve reflejada la práctica de los Orientadores y sus Departamentos en los cuadros referenciales que hemos observado? ¿Con qué intensidad? ¿Es más rica y diversa la práctica real que la teoría legal? ¿Cuál es realmente lo que se está haciendo en los IES?

El artículo 42 del Reglamento Orgánico de los Institutos de Educación Secundaria (R.D. 83/1996, de 26 de enero. BOE, 21-2-96), establece, con absoluta claridad, las funciones o tareas internas que han de asumir los Departamentos de Orientación. Estas funciones o tareas se pueden aglutinar, con carácter general, en torno a los ocho grandes ámbitos de intervención que presentamos en este segundo objetivo, aun cuando nos fijemos con interés en los tres que hemos señalado en el primer objetivo que hemos abordado. Pues, bien, veamos cómo se agrupan, en torno a estos ámbitos de intervención, el conjunto de las propuestas o tareas que actualmente están llevando a cabo los D.O. de los IES de Valladolid y su provincia, a fin de comprobar cuán cerca, o no, está la teoría de la práctica y la práctica de la teoría. Para ello, hemos elaborado la siguiente tabla de registro de datos referidos al conjunto total de las respuestas reflejadas en el cuestionario que hemos aplicado.

\section{Cuadro no 4}

REPERTORIO INDICATIVO DE LAS TAREAS DE LOS DEPARTAMENTOS DE ORIENTACIÓN DE LOS IES DE VALLADOLID Y SU PROVINCIA

\begin{tabular}{|l|l|l|l|l|l|l|l|}
\hline PAT & OAP & APEA & EPS & PDC & PGS & PEC & AIAF \\
\hline 26 & 25 & 24 & 25 & 16 & 9 & 9 & 11 \\
\hline $100 \%$ & $96 \%$ & $92 \%$ & $96 \%$ & $61 \%$ & $34 \%$ & $34 \%$ & $42 \%$ \\
\hline FOL & PABE & PAD & PASE & PPDR & PPDC & GTPPC & PI \\
\hline 4 & 3 & 20 & 1 & 1 & 2 & 1 & 9 \\
\hline $15 \%$ & $11 \%$ & $77 \%$ & $4 \%$ & $4 \%$ & $8 \%$ & $4 \%$ & $34 \%$ \\
\hline PHV & AC & EPSA & PD & AM & PME & PACC & TE \\
\hline 1 & 5 & 1 & 2 & 1 & 1 & 1 & 1 \\
\hline $4 \%$ & $19 \%$ & $4 \%$ & $8 \%$ & $4 \%$ & $4 \%$ & $4 \%$ & $4 \%$ \\
\hline PFP & PTPA & POE & ---------- & ---------- & ---------- & ---------- & ---------- \\
\hline 2 & 1 & 1 & --------- & ---------- & ---------- & ---------- & ---------- \\
\hline $8 \%$ & $4 \%$ & $4 \%$ & ---------- & ---------- & ---------- & ----------- & ---------- \\
\hline
\end{tabular}

Fuente: Elaboración propia. Curso 1998-99. Total de IES consultados, 31. Total de cuestionarios contestados, 26. 
REFERENCIAS:

1. PAT: PLAN DE ACCION TUTORIAL.

2. OAP: ORIENTACION ACADEMICO-PROFESIONAL.

3. APEA: APOYO AL PROCESO DE ENSEÑANZA-APRENDIZAJE.

4. EPS: EVALUACION PSICOPEDAGOGICA.

5. PDC: PROGRAMA DE DIVERSIFICACION CURRICULAR.

6. PGS: PROGRAMA DE GARANTIA SOCIAL.

7. PEC: PROGRAMA DE EDUCACION COMPENSATORIA.

8. AIAF: ATENCION INDIVIDUALIZADA AL ALUMNADO Y FAMILIAS.

9. FOL: FORMACION OCUPACIONAL LABORAL.

10. PABE: PROGRAMA DE ABSENTISMO ESCOLAR.

11. PAD: PROGRAMA DE ATENCION A LA DIVERSIDAD.

12. PASE: PROGRAMA DE ATENCION SOCIO-EDUCATIVA.

13. PPDR: PROGRAMA DE PREVENCION DE DROGODEPENDENCIAS.

14. PPDC: PROPUESTAS PARA EL DESARROLLO DEL CURRICULO.

15. GTPPC: GRUPO DE TRABAJO CON EL PROFESORADO DEL PRIMER CICLO.

16. PI: PROGRAMA DE INTEGRACION.

17. PHV: PROGRAMA DE HABITOS DE LA VIDA DIARIA.

18. AC: ADAPTACIONES CURRICULARES.

19. EPSA: PROGRAMA DE EDUCACION PARA LA SALUD.

20. PD: PROGRAMA DISCOVER.

21. AM: AULA MADURATIVA.

22. PME: PROGRAMA DE ATENCION A MINORIAS ETNICAS.

23. PACC: PROGRAMA DE ACOGIDA Y CONVIVENCIA EN EL CENTRO.

24. TE: TECNICAS DE ESTUDIO.

25. PFP: PROGRAMA DE FORMACION DEL PROFESORADO.

26. PTPA: PROGRAMA PARA EL TRATAMIENTO DE PROBLEMAS DE APRENDIZAJE.

27. POE: PROGRAMAS DE ORIENTACION ESCOLAR.

Algunas consideraciones sobre estos datos nos predisponen a fijarnos en aquellas tareas que, con mayor frecuencia, forman parte del quehacer cotidiano de los D.O. de los IES. En síntesis, y huyendo de cualquier posible interpretación casuística, concretaremos, de una manera global, el amplio repertorio de respuestas que hemos obtenido, y que pasamos a exponer brevemente, dejando al lector suficientes espacios abiertos para la reflexión y el comentario particular. Nosotros haremos un especial hincapié en las siguientes:

- Se constata que la totalidad de los D.O. (100\%), están implicados, aunque tal vez con distinta intensidad (depende del grado de compromiso de los tutores), en tareas de planificación, aplicación y evaluación de Programas de Acción Tutorial, dando, así, respuesta real a uno de los ámbitos de intervención y apoyo sugerido, reiterativamente, por las diferentes normativas que se han venido sucediendo en estos últimos seis años, y que hemos recogido globalmente en el cuadro no 3. Existe, pues, un claro y decidido apoyo al PAT (aunque con distintos matices según sus protagonistas: orientador, tutores) que se concreta en una serie amplia de acciones cuya relevancia se manifiesta, especialmente, en aquellas que se refieren a la atención y asesoramiento a los alumnos que lo solicitan, al apoyo técnico a tutores en la elaboración del material de acción tutorial, a la ayuda individualizada al alumnado y familias y a la elaboración de técnicas de trabajo intelectual. Consecuentemente, se puede decir que se da una aceptable 
sintonía entre lo normativo-teórico y lo descriptivo-institucional como se podrá comprobar a poco que nos fijemos en los cuadros $n^{\circ} 3$ y $n^{\circ} 5$ y realicemos un sencillo ejercicio de análisis comparativo.

\section{Cuadro no 5}

\section{CAMPO № 1: APOYO AL PLAN DE ACCION TUTORIAL}

1‥ APOYO TECNICO A TUTORES EN LA ELABORACION DEL MATERIAL DE ACCION TUTORIAL.

2‥ AYUDA Y ORIENTACION A LOS PADRES.

3‥ ATENCION Y ASESORAMIENTO A LOS ALUMNOS QUE LO SOLICITEN.

4‥ ACTIVIDADES DE CONVIVENCIA E INTEGRACION DEL GRUPO.

5‥ ACTIVIDADES DE MEJORA DE LA AUTOESTIMA.

6 . ACTIVIDADES DE EDUCACION PARA EL CONSUMO.

7a. ACTIVIDADES PARA EL FOEMENTO DE LA IGUALDAD EN LA DIFERENCIA.

8. PROGRAMAS DE ATENCION MANTENIDA EN TAREAS.

9a. PROGRAMAS DE HABILIDADES SOCIALES.

10.

11‥ REUNIONES PARA EL SEGUIMIENTO Y EVALUACION DEL PAT.

- Con unos niveles de compromiso y dedicación semejantes, los D.O. consultados certifican que están llevando a término dos tipos de acciones relevantes, como son: la orientación académica y profesional (96\%) y el apoyo al proceso de enseñanza y aprendizaje (92\%), dentro del cual adquiere especial relevancia la evaluación psicopedagógica (96\%). Así se desprende de los datos volcados en la tabla que hemos configurado en el cuadro no 4 . No obstante, en aras a puntualizar y clarificar qué tipo de actuaciones se están generando y desarrollando desde los Departamentos, referidas al apoyo al proceso de enseñanza y aprendizaje, hemos de mencionar aquellas que con mayor asiduidad se vienen sucediendo habitualmente y forman parte integrante de la labor diaria de los orientadores $y$, en ocasiones, de algunos tutores. Así, tenemos que referirnos a las que apuntan a la prevención, detección e intervención psicopedagógica con los alumnos que presentan problemáticas en las materias más relevantes de su nivel; al diseño y evaluación de programas de diversificación curricular, de atención a la diversidad, de garantía social y de educación compensatoria; a la ayuda didáctico-pedagógica de aquellos profesores que tienen en sus aulas alumnos con necesidades educativas especiales; al asesoramiento a los distintos componentes de la comunidad escolar en cuestiones específicas referidas a los niveles de planificación del Centro y otros, como son: PEC (Proyecto Educativo de Centro, PCE (Proyecto Curricular de Etapa), PA (Programación de Aula), PEI (Programa de Enriquecimiento Instrumental), ACls (Adaptaciones Curriculares Individuales), PDI (Programas de Desarrollo Individual); a las tareas de colaboración con la Comisión de Coordinación Pedagógica en la elaboración de los documentos oficiales; a las acciones de apoyo que se proyectan hacia la orientación y refuerzo de los acnees (alumnos con necesidades educa- 
tivas especiales) y su inclusión en contextos sociales y académicos; a la evaluación psicopedagógica del alumnado en colaboración con sus profesorestutores correspondientes.

\section{Cuadro no 6}

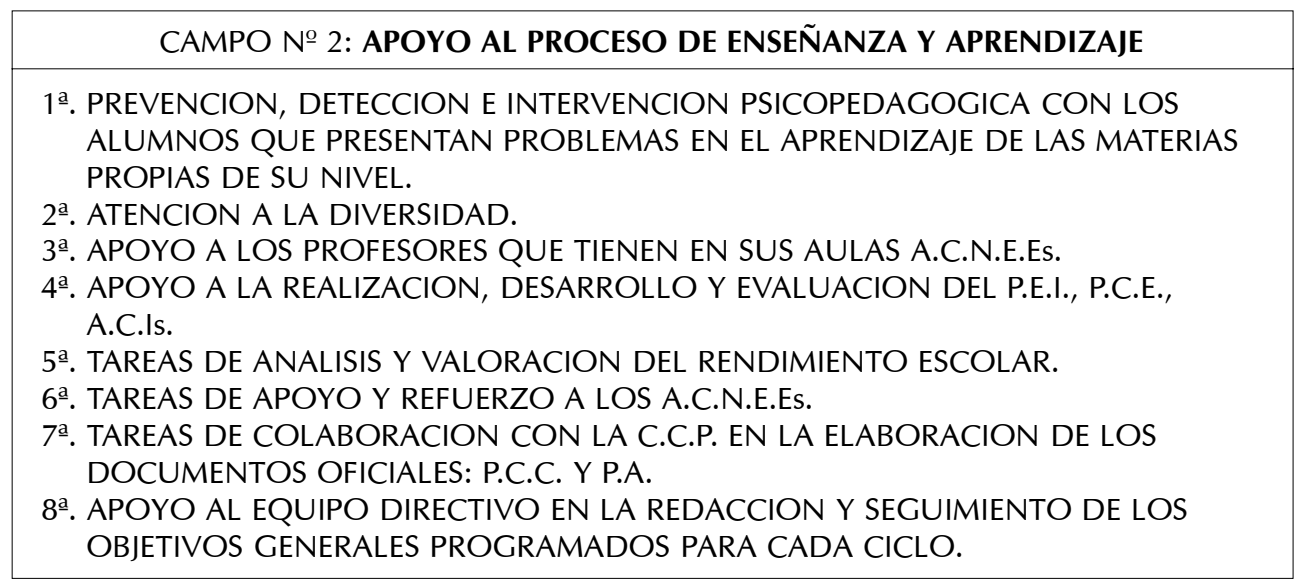

Nuevamente observamos un acercamiento considerable entre la teoría y la acción, aunque este tipo de convivencia se manifieste, a menudo, con diferentes grados de intensidad y eficiencia orientadora, por cuanto que, en no pocas ocasiones y contextos, suele ser más espectacular lo que se dice que se pretende hacer que lo que realmente se consigue haciendo, es decir, que como dice el refranero, "a veces es más el ruido que las nueces".

Ahora vamos a dirigir nuestra atención hacia el tercero de los ámbitos de actuación, cual es el que enlaza con el apoyo al plan de orientación académica y profesional. Situados en este campo, nos percatamos del valor que se le concede, fundamentalmente, por su relevancia en la práctica totalidad de los IES como queda demostrado a través de manifestaciones y actuaciones de diferente rango que hemos recogido en el cuadro $n^{\circ}$ 7. En el estudio que hemos realizado, constatamos la existencia de una serie de actuaciones funcionales mediante las cuales se presta orientación directa a los alumnos que van a realizar los programas de Diversificación Curricular y Garantía Social; orientación y asesoramiento personalizado a los alumnos que continúan en el sistema educativo no universitario cursando los estudios de Bachillerato o Ciclos Formativos, o están en vías de acceder a la universidad para el estudio de carreras concretas; información y consejo para la elección de asignaturas optativas e itinerarios educativos; ayuda para facilitar la toma de decisiones; información y asistencia sobre las posibles salidas académicas y profesionales y sobre el mundo laboral; realización de visitas a distintos centros universitarios para conocer sus planes de estudios, la organización académica, salidas profesionales, etc., mediante conferencias o charlas programadas en función de los intereses y preferencias de los alumnos. 


\section{Cuadro no 7}

\section{CAMPO № 3: APOYO AL PLAN DE ORIENTACION ACADEMICA Y PROFESIONAL}

1‥ INTERVENCION DIRECTA CON LOS ALUMNOS DE $2^{\circ}$ Y $4^{\circ}$ DE E.S.O., A TRAVES DE PRUEBAS PSICOPEDAGOGICAS.

2‥ ORIENTACION DIRECTA A LOS ALUMNOS QUE VAN A REALIZAR EL PROGRAMA DE GARANTIA SOCIAL.

3‥ ORIENTACION DIRECTA A LOS GRUPOS DE DIVERSIFICACION CURRICULAR.

4‥ ASESORAMIENTO PERSONALIZADO A LOS ALUMNOS QUE VAN A CURSAR EL BACHILLERATO O CICLOS FORMATIVOS.

5‥ ASESORAMIENTO EN LA ELECCION DE ASIGNATURAS OPTATIVAS E ITINERARIOS EDUCATIVOS.

6. AYUDA EN LA TOMA DE DECISIONES.

7a. ACTIVIDADES DE AUTOCONOCIMIENTO, DE INFORMACION DEL MERCADO LABORAL Y DE ENTRENAMIENTO EN TOMA DE DECISIONES.

8‥ INFORMACION SOBRE SALIDAS ACADEMICAS Y PROFESIONALES.

También en este ámbito de actuación, los D.O. cumplen con las expectativas que sugiere la literatura normativa que se ha ido gestando y desarrollando en los últimos años. Sin embargo, echamos en falta un cierto compromiso de los tutores en tareas de elaboración, implementación y evaluación del POAP (Plan de Orientación Académica y profesional), lo que, desde nuestro punto de vista, perjudica considerablemente la confección de planes de actuación y programas de orientación académica y profesional, ya que con frecuencia estas acciones se reducen a intervenciones o sesiones eminentemente informativas y de marcado carácter puntual. Desaparece, pues, la perspectiva de "programa" y, por consiguiente, su necesaria inclusión en el PCE (Proyecto Curricular de Etapa) así como la posibilidad de evaluar los logros alcanzados, las dificultades encontradas, los factores influyentes o condicionantes en ambos casos, los aspectos a modificar o reformular, la propia validez del programa, las tareas realizadas por los alumnos, la responsabilidad adquirida por los educadores comprometidos, etc.

Una vez hemos finalizado el comentario que nos sugieren los indicadores más relevantes que hemos abordado con formato de "ámbitos de intervención" de los D.O., y a pesar de estar situados en el camino correcto, aunque aún falte un amplio trayecto por recorrer en el campo de la orientación psicopedagógica, se impone explicitar, además de estas tareas, otras de necesaria inclusión en la oferta orientadora de los IES. Es el caso de los programas de carácter preventivo y/o anticipador que abordan problemáticas muy actuales que afectan al alumnado, a la formación del profesorado $y$, consecuentemente, a la innovación educativa y a la calidad de la enseñanza, y que, de alguna forma, están en la mente y hasta en la práctica de algunos orientadores. Nos estamos refiriendo a propuestas temáticas relacionadas con cuestiones tan actuales como: alcoholismo, drogadicción, racismo, atención socioeducativa, técnicas de mejora del rendimiento escolar, programas de convivencia y acogida en el Centro, educación para la salud, educación para el consumo, formación del profesorado, investigación educativa, etc. Todas estas propuestas, dependiendo de su contenido, se hallan incluidas en los cuatro campos de apoyo que nos resta por 
comentar: apoyo a la atención a la diversidad; apoyo a la investigación e innovación educativa; apoyo a la evaluación psicopedagógica; apoyo y orientación a la familia; apoyo a la formación del profesorado. Nos referiremos, brevemente, a los resultados obtenidos en el estudio objeto de comentario en este artículo, su grado de representatividad en el conjunto de las tareas y su indicador de ajuste a la propuesta-modelo sugerida por el MEC.

Así, pues, el cuarto ámbito o campo de actuación, apoyo a la atención a la diversidad,(conjunto de medidas específicas que afectan a la integración escolar, educación especial y educación compensatoria) se concreta en no pocas acciones que se están llevando a cabo en un considerable número de D.O. (en el 77\%), como hemos comprobado en esta investigación: intervenciones con alumnos de $1^{\circ}$ y $2^{\circ}$ de ESO que precisan atención especial, como es el caso de las minorías étnicas; seguimiento de los informes elaborados por los EOEPs (Equipos de Orientación Educativa y Psicopedagógica) y de los programas de diversificación curricular (61\%), especialmente destinados a alumnos de $3^{\circ}$ y $4^{\circ}$ de ESO; apoyo a la elaboración de programas de Garantía Social (34\%); apoyo y orientación al profesorado para la elaboración de las ACls para alumnos de $1 \stackrel{\circ}{\circ} 2^{\circ}$ y $3^{\circ}$ de ESO $(19 \%)$, y otras acciones que recogemos en el cuadro adjunto $\mathrm{n}^{\circ} 8$.

\section{Cuadro no 8}

CAMPO № 4: APOYO A LA ATENCION A LA DIVERSIDAD

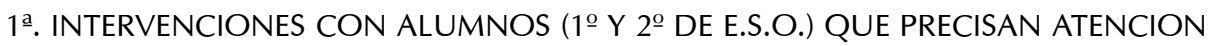
ESPECIAL: A.C.N.E.Es., MINORIAS ETNICAS, ALUMNOS CON DIFICULTADES IMPORTANTES DE APRENDIZAJE, A TRAVES DE PROGRAMAS ESPECIFICOS.

2ª SEGUIMIENTO DE LOS INFORMES ENVIADOS POR LOS E.O.E.Ps.

3‥ SEGUIMIENTO Y VALORACION DE LOS PROGRAMAS PARA LOS A.C.N.E.Es. Y LOS ALUMNOS DE DIVERSIFICACION CURRICULAR ( $3^{\circ}$ Y $4^{\circ}$ DE E.S.O.).

4‥ APOYO EN LA ELABORACION DE LOS PROGRAMAS DE GARANTIA SOCIAL (ALUMNOS MAYORES DE 16 AÑOS).

5‥ ENTREVISTAS CON LAS FAMILIAS Y DEVOLUCION DE DATOS.

6. TOMA DE DECISIONES SOBRE LA PROMOCION, O NO, DE LOS A.C.N.E.Es. AL CICLO SIGUIENTE.

7ª. APOYO Y ORIENTACION AL PROFESORADO PARA LA ELABORACION DE LAS A.C.Is ( $1^{\circ}, 2^{\circ}$ Y $3^{\circ}$ DE E.S.O.).

8․ ORIENTACION EN LA ELECCION DE LA OPTATIVIDAD CURRICULAR.

El quinto ámbito de actuación lo describimos con el nombre de apoyo a la investigación e innovación educativa. Desde aquí, resaltamos las tareas más relevantes en las que se ven inmersos algunos profesores-tutores, por sugerencia de los Orientadores de los IES, en beneficio de su propia formación y de la de sus alumnos: participación en proyectos de innovación educativa, colaboración en trabajos de investigación, realización de cursos ofertados por la Dirección Provincial a través de los CPRs (8\%), etc. No obstante, se plantea con urgencia la ineludible necesidad de un cambio gradual y progresivo en la mentalidad y actitudes del profesorado, en aras 
a desarrollar y practicar en los Centros una cultura pedagógica que apueste radicalmente por la reflexión sobre la práctica, por la innovación, por la mejora de una oferta educativa constantemente renovada, por la construcción y reelaboración del conocimiento, por querer, simplemente, cambiar a mejor.

\section{Cuadro no 9}

\section{CAMPO № 5: APOYO A LA INVESTIGACION E INNOVACION EDUCATIVA}

1‥ PARTICIPACION EN LOS PROYECTOS DE INNOVACION EDUCATIVA CONVOCADOS POR LA JUNTA DE CASTILLA Y LEON, O POR EL MEC.

2a. PARTICIPACION EN PROGRAMAS DE INVESTIGACION EN COLABORACION CON LA UNIVERSIDAD (FACULTAD DE EDUCACION).

3a. REALIZACION DE CURSOS A TRAVES DE LOS C.P.Rs.

4‥ REALIZACION DE TRABAJOS EN GRUPO CON PROFESORES DEL CENTRO, ORIENTADORES, O.E.Gs.

El sexto campo de acción se refiere al apoyo a la evaluación psicopedagógica (96\%). Aun cuando ya nos hemos acercado a él, aquí lo hacemos con mayor amplitud y abundancia de actuaciones. En este sentido, los Departamentos de Orientación de los IES están desarrollando funciones relacionadas con la recogida de información de profesores, tutores, padres y del propio alumno en orden a poseer un conocimiento mucho más cercano y real; tareas de observación en el aula, aplicación de pruebas específicas, realización de entrevistas, elaboración de informes, etc. Esta parcela, tal vez, sea una de las mejores atendidas en los IES, lo cual no quiere decir que lo que se está haciendo es suficiente, ni que la colaboración de los tutores sea la idónea. Los Orientadores esperan mucha más implicación de los docentes afectados, también de los profesores especialistas e incluso de los Equipos Directivos que, a veces, no interpretan con suficiente claridad estos mensajes.

\section{Cuadro no 10}

\section{CAMPO № 6: APOYO A LA EVALUACION PSICOPEDAGOGICA}

1‥ RECOGIDA DE INFORMACION DE TUTORES, PROFESORES, PADRES Y DEL PROPIO ALUMNO.

2‥ REALIZACION DE LA EVALUACION PSICOPEDAGOGICA PRECEPTIVA: ALUMNOS DE DIVERSIFICACION CURRICULAR, ALUMNOS DE GARANTIA SOCIAL.

3a. REALIZACION DE LA EVALUACION PSICOPEDAGOGICA DE LOS ALUMNOS OBJETO DE INTERVENCION Y APOYO ESPECIAL EN COLABORACION CON EL PROFESOR TERAPEUTA: A.C.N.E.ES., ALUMNOS INCLUIDOS EN PROGRAMAS DE EDUCACION COMPENSATORIA.

4‥ TAREAS DE OBSERVACION EN EL AULA, REVISION DE TRABAJOS ESCOLARES, APLICACION DE PRUEBAS ESPECIFICAS, REALIZACION DE ENTREVISTAS, ETC.

5‥ ELABORACION DE INFORMES DE EVALUACION PSICOPEDAGOGICA. 
El séptimo, apoyo y orientación a la familia (42\%), es un ámbito muy interesante pero, actualmente, a nuestro entender, insuficientemente trabajado y muy necesitado de atención preferente. La investigación pone de manifiesto las actividades, un tanto generales e inespecíficas, que se están realizando desde los D.O.: asesoramiento individualizado a las familias que lo solicitan; actividades de información sobre el POAP; sesiones para implicar a los padres en el proceso educativo de sus hijos; actividades de formación educativa, etc. En ocasiones, estos encuentros (orientador-padres / tutorpadres) se producen esporádicamente y, casi siempre, para abordar cuestiones relativas a la evaluación de los aprendizajes o relacionadas con el comportamiento conductual en el Centro. Asimismo, se observa la ausencia de planes o, cuanto menos, de actividades de acción formativa que redunden en beneficio de una mejor y más actualizada formación de los padres y, al mismo tiempo, de la educación que han de facilitar a sus hijos. En consecuencia, se debería potenciar mucho más la creación de escuelas de padres (desde $1^{\circ}$ de la ESO) por cuanto que la familia ,y el estilo educativo que comparte a diario con los hijos, constituye una pieza irremplazable del complicado puzzle de la educación, en el que la labor de la orientación y tutoría que se oferta en los IES no es ni sustitutiva ni suficiente de aquella, simplemente es complementaria y, si se prefiere, más técnica.

\section{Cuadro no 11}

CAMPO № 7: APOYO ORIENTACION A LA FAMILIA

1‥ COLABORACION CON EL EQUIPO DIRECTIVO EN LA PREPARACION DE LA REUNIONES DE PRINCIPIO Y FINAL DE CURSO.

2a. INTERVENCIONES INDIVIDUALES CON LAS FAMILIAS DESTINADAS A PROPORCIONARLAS LA INFORMACION Y EL ASESORAMIENTO QUE PRECISEN.

3 a . REUNIONES COLECTIVAS ENTRE TUTORES Y PADRES; ENTRE PADRES Y DEPARTAMENTO DE ORIENTACION, PARA INFORMARLES DEL PLAN DE ORIENTACION ACADEMICA Y PROFESIONAL Y AYUDARLES EN LA TOMA DE DECISIONES DE SUS HIJOS.

4‥ REUNIONES PERIODICAS CON LAS FAMILIAS AL OBJETO DE IMPLICARLAS EN EL PROCESO EDUCATIVO DE SUS HIJOS.

5 a. ACTIVIDADES FORMATIVAS.

Por último, el octavo campo, es decir, el apoyo a la formación del profesorado, (8\%) tanto desde el propio IES (muy deseable, siempre que sea posible, y altamente enriquecedor para el conjunto del profesorado copartícipe) como a través de otras instancias o instituciones reconocidas, como es el caso de los CPRs, Facultades de Educación, etc. Sin duda alguna, es ésta una faceta del curriculum vitae de los profesionales, poco ajustada, generalmente, a dimensiones didácticas y psicopedagógicas $y$, por el contrario, prioritariamente atendida en sus dimensiones afines al objeto propio de la disciplina que imparten en sus aulas, casi siempre que se trata de elegir o seleccionar, voluntariamente, cursos de formación permanente. Ambas, conjuntamente, deberían constituir o conformar las dos caras de una misma moneda de uso legal. Lo cierto es que de los veintiséis IES de los que tenemos información, solamen- 
te en dos ha habido profesores que han estado implicados en programas o actividades de formación impartidas o desarrolladas en el propio IES, lo cual no quiere decir, ni mucho menos, que no pocos profesores hayan podido optar por otras vías de formación extracentro. No obstante, hemos de reconocer su generosa participación, como tutores, en el practicum de la formación de los alumnos del CAP (Curso de Aptitud Pedagógica).

\section{Cuadro no 12}

\begin{tabular}{|l|}
\hline CAMPO № 8: APOYO A LA FORMACION DEL PROFESORADO \\
\hline 1․ PROGRAMA DE PRACTICAS DE FORMACION PARA LOS ALUMNOS DE 5o DE PSI- \\
COPEDAGOGIA (FACULTAD DE EDUCACION). \\
2a. PROGRAMA DE PRACTICAS DE FORMACION PARA LOS ALUMNOS DEL CURSO \\
DE APTITUD PEDAGOGICA (C.A.P.). \\
3a. COORDINACION DE SEMINARIOS Y GRUPOS DE TRABAJO SOBRE:DISEÑO \\
CURRICULAR, ORGANIZACION, TRANSVERSALIDAD. \\
4. SUGERENCIAS Y ORIENTACION AL PROFESORADO SOBRE LOS CURSOS MAS \\
CONVENIENTES PARA SU FORMACION OFERTADOS POR LOS CENTROS DE PRO- \\
FESORES Y RECURSOS (C.P.RS.).
\end{tabular}

En el estudio que estamos comentando, hemos recogido, como se puede ver en el cuadro $\mathrm{n}^{-} \mathbf{1 2}$, lo que realmente se viene realizando en consonancia con aspectos que aluden, con claridad, a la formación permanente del profesorado y sus acciones continuas de perfeccionamiento o enriquecimiento psicopedagógico y didáctico, y que, sin embargo, aquí no se constatan como tales.

Junto a su participación en seminarios y grupos de trabajos, normalmente sobre diseño curricular y transversalidad, colaboran en otra serie de actividades (no formativas en sentido estricto) y suelen pedir información a los orientadores sobre cursos ofertados por los CPRs.

Los orientadores, con frecuencia, se quejan de la poca preparación del profesorado en aspectos didácticos, metodológicos y psicopedagógicos, bien porque no forman parte del currículo de sus carreras universitarias, bien porque no se muestran convencidos de su imprescindible utilidad profesional y educativa, bien porque no se ha reflexionado lo suficiente sobre la Reforma, en general, y más concretamente en sus artículos 55 y 60 de la LOGSE, en los que se recogen, por un lado, una serie de factores que favorecen la calidad y mejora de la enseñanza, como por ejemplo, la cualificación y formación del profesorado, la innovación y la investigación educativa, entre otros, y, por otro, que la tutoría y la orientación de los alumnos forma parte de la función docente, lo que, sin duda alguna, supone, o debería suponer, un cambio radical en ciertas actitudes latentes, al menos, en una parte del profesorado para quienes instruir sigue siendo más importante que educar aunque se sepa que se educa orientando y se orienta educando.

Además de este amplio y complejo cúmulo de "quehaceres de apoyo" en los que los orientadores, con más o menos intensidad y variedad, se encuentran implicados, 
realizan actividades propiamente docentes a las que suelen dedicar, como media, entre ocho y diez horas semanales. Las asignaturas (psicología, ética) y los distintos programas que imparten, siempre en función de la realidad de cada I.E.S, se aglutinan con cierta lentitud y siempre atendiendo a criterios de conveniencia pedagógica. Entre estos, hemos de referirnos a los programas de transición a la vida adulta y activa; a los programas de aprender a pensar; de educación para la salud (4\%); de educación para la convivencia; de acogida y convivencia en el Centro, (4\%); de atención a las minorías étnicas (4\%); de hábitos de la vida diaria (4\%); de prevención de drogodependencias (4\%); de tratamiento de los problemas de aprendizaje (4\%); discover (8\%); de absentismo escolar (11\%); de formación ocupacional (15\%) y de educación compensatoria (34\%), entre otros.

5.3. Objetivo $n^{\circ}$ 3: "Reflexionar, críticamente, sobre el marco institucional normativo (documentos legales) y práctico descriptivo (lo que se está haciendo en los Centros)..."

Nuestra intención no es otra que elaborar y suscribir una respuesta fundamentada en las intervenciones reales que, en estos momentos, después de seis años de funcionamiento de los D.O., se vienen implementando en los I.E.S., con especial referencia al caso de Valladolid y su provincia, y en las sugerencias formuladas en la literatura oficial (Leyes, Reales Decretos, Resoluciones, Circulares, etc), a las que ya nos hemos referido al inicio del artículo.

Pues, bien; se trata de hacer una valoración comparativa entre los ámbitos de actuación y funciones de intervención de los D.O. de los I.E.S.; todo ello desde el marco institucional normativo, es decir, según la norma o normas reguladoras (R.D. 929/1993 de 18 de junio; R.D. 83/1996, de 26 de enero; otras de distinto rango administrativo) y lo que realmente se está trabajando en los escenarios de la práctica orientadora diaria, como es el caso que nos ocupa.

Observando el plan de actividades propuesto por el MEC a los D.O., hemos de concluir que, en lo esencial, difiere muy poco de lo que realmente se está trabajando en los I.E.S. Pero más aún; hay que decir, con absoluta claridad, que en algunos ámbitos de apoyo (Apoyo al proceso de enseñanza-aprendizaje, entre otros), lo que es normativo se ve ampliamente superado por lo descriptivo; buena prueba de ello lo constituye el repertorio indicativo de las veintiséis tareas (con valoraciones diferentes) en las que están implicados dichos Departamentos. No obstante, conviene recordar, entre otras, las cuestiones que vamos a exponer, a efectos de clarificar, un poco más, la respuesta o respuestas que demanda el comentario del objetivo que estamos intentado abordar en su fase de valoración final. A este respecto hemos de valorar:

- Las tareas implementadas, con carácter general, desde y por los D.O. y sus responsables más directos (orientadores), que hemos insertado en ocho grandes ámbitos de intervención y apoyo, y que responden, en su conjunto, satisfactoriamente, a las expectativas formuladas por un amplio combinado de IES. al referirse a los tres primeros ámbitos de actuación ya comentados como respuesta al objetivo nำ1. 
- Siendo todas ellas suficientemente importantes, merecen, a nuestro juicio, especial consideración aquellas que se agrupan en torno a los apoyos al plan de acción tutorial, al proceso de enseñanza-aprendizaje, al plan de orientación académica y profesional, a la evaluación psicopedagógica, a la atención a la diversidad, a la atención individualizada al alumno y al apoyo a la integración escolar.

- Por el contrario, los ámbitos de intervención orientadora y apoyo educativo menos favorecidos son los que afectan a la investigación e innovación educativa, a la orientación familiar y a la formación del profesorado, a nuestro juicio muy importante, porque sin formación no es posible la innovación. No obstante, hemos de decir que se está avanzando considerablemente, aunque no siempre con la prontitud deseable, si comparamos lo poco que se ha hecho en este campo, históricamente poco protegido, con los logros obtenidos en el momento actual, aun cuando la LOGSE, con diez años de vigencia, constituya, todavía, una propuesta educativa por desarrollar en no pocos de sus planteamientos psicopedagógicos y curriculares.

- El papel que se otorga, en general, al conjunto de las actuaciones dirigidas a que los alumnos desarrollen las capacidades implicadas en el proceso de toma de decisiones, de tal manera que conozcan y valoren, de forma ajustada, sus propias capacidades, motivaciones e intereses.

- La relevancia de las distintas actuaciones desarrolladas por los Orientadores en el campo, siempre complejo, del diseño curricular en sus diversas opciones educativas.

- El alto grado de compromiso, por parte de los Orientadores para llevar a cabo diversas acciones que permiten iniciar y mantener, progresivamente, una comunicación fluida con las familias, tanto para intercambiar informaciones sobre aspectos que pueden resultar relevantes para mejorar el proceso de aprendizaje de los alumnos, como para orientarles y promover su cooperación con el profesorado.

Otros logros, ciertamente descollantes y oportunos, se pueden concretar, de manera abreviada, en:

- Difusión generalizada de la LOGSE entre los profesores de los diferentes Departamentos Didácticos, especialmente de aquellos aspectos relacionados con la atención a la diversidad y sus campos de intervención: alumnos con necesidades educativas especiales (permanentes y/o transitorias); alumnos incluidos en programas de Diversificación Curricular y alumnos de los programas de Garantía Social.

- Progresivo aumento en el reconocimiento de la importancia, necesidad y permanente presencia de los D.O. en todos los Centros de Educación Secundaria (aunque con ciertas reservas), como un instrumento útil al servicio de toda la comunidad educativa.

- Paulatina inserción del D.O. en el organigrama organizativo, psicopedagógico y didáctico de los I.E.S., eliminando, poco a poco, el rechazo (no explícito) que inicialmente tuvo en algunos Centros, y borrando de algunas mentes la falsa idea 
por la que los Orientadores eran considerados poco menos que "invasores" y representantes del M.E.C.

- Eliminación de concepciones erróneas acerca del rol de los D.O., desterrando la idea de "cajón de sastre", en favor de otras más en la línea de la Reforma: eje transversal y favorecedor del proceso educativo-formativo en el ámbito de una escuela más comprensiva y diversa.

5.4. Objetivo $n^{\circ}$ 4: "A la vista de los resultados obtenidos, formular propuestas y recomendaciones de mejora para..."

Por último, y a modo de conclusiones finales, ¿cuáles son los temas que, por su relevancia e interés organizativo, educativo, etc., demandan una mayor atención y continuidad y que, lógicamente, habría que abordar con urgencia? Los Orientadores consideran prioritarios los siguientes:

- Potenciar los D.O. a través de un apoyo más decidido, explícito y eficaz por parte del M.E.C. (actualmente por la Junta de Castilla y León) y de la Dirección Provincial, atendiendo a razones técnicas (incrementar, en algunos casos, la dotación del profesorado especialista, concretamente el que se refiere a los ámbitos sociolingüístico, científico y tecnológico) y económicas (aumentar el presupuesto del Centro y destinar una parte del mismo al D.O. para la adquisición de material de testing, informático, didáctico, etc.).

- Acrecentar y desarrollar, más eficazmente, la coordinación entre el D.O. y los Departamentos Didácticos, entre aquél y el Equipo de Tutores, no solamente en las tareas que les son encomendadas por la norma, sino, también, en aquellas otras propias de cada contexto escolar, que reclaman su atención y colaboración. Lo importante es encontrar siempre una respuesta comunitaria a las exigencias que plantea el ejercicio de la acción orientadora.

- Prestar más atención, desde una perspectiva fundamentalmente terapéutica, a los alumnos que sin estar integrados en ningún tipo de programa específico, presentan problemas de aprendizaje fácilmente subsanables.

- Desarrollar y potenciar, desde el Centro, acciones concretas de orientación familiar.

- Mejorar el apoyo y la atención a la diversidad desde planteamientos organizativos, didácticos, metodológicos y de trabajo colaborativo en grupo, mucho más eficaces.

- Potenciar la transmisión de información, ayuda y apoyo técnico a los Tutores y, desde estos, al resto del profesorado.

- Iniciar, apoyar e intervenir, desde el consenso, en la formación didactico-pedagógica del profesorado de los I.E.S mediante acciones desarrolladas en el propio Centro.

- Mejorar el marco organizativo establecido en la LOGSE para los D.O., dotándoles de más personal cualificado (trabajadores sociales, profesores de audición y lenguaje), para poder responder, más eficazmente, a las múltiples y variadas 
necesidades que, continuamente, se generan dentro de un proyecto amplísimo $y$, a veces, con pocos medios.

- Reducir a la mitad, que no suprimir, las horas semanales de los Orientaciones dedicadas a la docencia que, en estos momentos, suelen ser unas ocho horas, como media, para aumentar la dedicación al Departamento y, consecuentemente, a los alumnos, padres y profesores.

- Establecer líneas de coordinación y colaboración recíproca entre los D.O. de los I.E.S. y los Equipos de Orientación Educativa y Psicopedagógica de los distintos sectores en los que están ubicados los Colegios de Educación Primaria "adscritos" a dichos institutos, a efectos de tener un conocimiento y, en su caso, una continuidad, mucho más individualizada, personalizada y enriquecedora con todos los alumnos que comienzan la ESO y, especialmente, con aquellos que presenten ciertas minusvalías y necesiten atenciones más especializadas.

- Trabajar cooperativamente con otros I.E.S. del sector al objeto de compartir experiencias, fomentar investigaciones, potenciar la renovación e innovación educativa y favorecer la calidad y mejora de la enseñanza.

- Eliminar las evidencias que se constatan en bastantes IES, mediante las cuales se puede comprobar, con preocupación, la insatisfacción de ciertos profesores con el trabajo que realizan los D.O. y, a su vez, la de los Orientadores que se quejan de la poca colaboración que encuentran en el profesorado a la hora de llevar a término programas, planes o acciones grupales de orientación educativa.

Estos son los temas que, actualmente, más preocupan a los Orientadores de los I.E.S. de Valladolid y provincia. A unos Orientadores que prestan sus servicios en Centros excesivamente poblados (todos, salvo puntuales excepciones, superan ampliamente los quinientos alumnos) y exigentes en la atención que reclaman sus alumnos. Sin embargo, estos Orientadores poseen, en general, una serie de ventajas que les permiten enfrentarse a un trabajo ya conocido, de antemano, en muchas de sus facetas más técnicas y específicas por haber estado, previamente, ejerciendo su profesión en Equipos de Orientación.

El hecho de que una gran mayoría de los Orientadores hayan Ilegado al Departamento después de haber dedicado los primeros años de su vida profesional (una media de diez años) al ejercicio del magisterio en las especialidades de Educación Especial y/o Audición y Lenguaje, o bien como integrantes de los Equipos Sectoriales de Orientación, supone haber recorrido todo un camino cargado de ricas experiencias y, consiguientemente, estar en el momento presente suficientemente sensibilizados con la temática propia de su quehacer diario, a la vez que mucho más cercanos a la problemática del alumnado y, por ende, a la oferta de orientación y de apoyo que necesitan.

\section{Propuestas de futuro}

Finalmente, y yendo un poco más allá del comentario que sobre las conclusiones del estudio que hemos realizado acabamos de presentar en sus aspectos más significativos y relevantes para los orientadores y tutores actuales y futuros, no quisiéramos 
dejar sin respuesta la siguiente pregunta: ¿qué retos y expectativas se plantean de cara a un futuro tan próximo como necesario? La respuesta, en parte, ya la hemos ofrecido. Sólo nos resta hacer hincapié en una serie de ideas claves y sencillas sugeridas desde la propia investigación, desde la teoría gestada, desde el camino histórico recorrido y desde la propia actividad práctica.

1‥ La realidad cotidiana de los D.O., con sus aciertos y errores, ha de poner especial interés en el debate y en la reflexión de lo que se está haciendo y cómo se está haciendo, sin reducir el margen de las ilusiones y de las nuevas propuestas.

2a . La mejora de la calidad de la coordinación (nivel de convergencia) entre el D.O. y los Departamentos Didácticos, como poderosos instrumentos de creación de riqueza educativa, ha de llevar a unos y a otros a la aceptación conjunta de nuevas responsabilidades y al desarrollo pleno de un nuevo estilo orientador.

$3^{a}$. Hay que seguir trabajando, con mayor fuerza si cabe, por la construcción de un verdadero espacio para la Orientación Psicopedagógica en los I.E.S., lo que supone potenciar el trabajo sectorial común para unificar aspectos de intervención y planes conjuntos de actuación.

4‥ Es necesario clarificar los andamios de la arquitectura orientadora que, para ser eficaz, deberá ser centralizada en lo funcional (a nivel de sector), y totalmente autónoma en lo esencial (a nivel de Centro).

5‥ Hay que entender que la docencia, por importante que sea ,y ciertamente lo es, no puede seguir interpretándose como el único quehacer que justifique todas y cada una de las responsabilidades y funciones de los profesores, en general, y de los tutores, en particular. La reflexión sobre la práctica, la investigación, la formación continua en aquellas parcelas psicopedagógicas y curriculares y la innovación didáctica, deben preceder, acompañar y seguir a la docencia.

$6^{\mathrm{a}}$. En adelante, una de la funciones propias del Orientador con respecto a los tutores y profesores ha de ser la de "formador de formadores", mediante la prestación de ayuda seleccionada y cualificada, aportando principios, técnicas e instrumentos apropiados a la tutoría y a la orientación de los alumnos y familias en su irrenunciable actividad educativa para la formación humana y para la resolución de problemas académicos y de toma responsable de decisiones.

7a. Se impone superar el modelo comparativo y, con él, el recurso fácil y cómodo de"cualquier tiempo pasado fue mejor", en favor de la creación de un espacio pedagógico y discursivo vinculado con la realidad de los IES, apto para la sensibilización y formación del profesorado.

$8^{\text {a }}$. Se hace necesario repensar la oferta horaria de los IES y, tal vez, su propio esquema organizativo poco flexible, en algunos casos, contemplando momentos para las reuniones, seminarios, grupos de trabajo, etc., sin que para ello se tengan que buscar huecos ocasionales fuera de la jornada de trabajo, lo que sin duda alguna es un refuerzo negativo para el tan necesitado trabajo cooperativo y en equipo. 
9a. Es importante resaltar el compromiso de vinculación que debe existir entre el desarrollo de la función tutorial y su proyección bimodal, evaluación-orientación psicopedagógica.

10 . Hay que ampliar las dimensiones del objeto de la orientación para que su referente no sea casi siempre y solamente el alumno y, por el contrario, casi nunca el profesor y el contexto en el que se pueden producir fallos y dificultades. En este sentido, y sin olvidar ningún tipo de intervención (entre otras, la intervención directa con los alumnos), es necesario potenciar la intervención a través de agentes educativos intracentro dirigiéndose más hacia la propia institución escolar, con una participación más intensa en referencia a los objetivos educativos, a la metodología, a los recursos y, en definitiva, colaborando en la formación permanente y en el apoyo al profesorado en sus pluriactuaciones.

11‥ Una constante que hay que abordar de cara a las actuaciones de los D.O. y que mejora sus propios aspectos organizativos, estructurales y de funcionamiento, es el tratamiento de la orientación a través de la introducción de programas de intervención (fundamentados en modelos de orientación), integrados en el currículo.

$12^{\mathrm{a}}$. Es cierto que todos los IES contemplan en su estructura organizativa la disposición de un sistema tutorial; pero no es menos cierto que esto lo hacen a unos niveles de competencia profesional (tutores) poco o casi nada especializados, por lo que habrá que potenciar la formación de sus agentes en este campo de conocimiento específico. Y es que los recursos humanos de los D.O. constituyen un elemento esencial que debe cuidarse dada la multiprofesionalidad de los equipos que los integran.

13a . En este momento, y como síntesis de todo lo expuesto, las grandes ideas que han de impulsar el desarrollo de los D.O, deberán ser: el compromiso libre del profesorado para con la atención y apoyo a la comprensividad de la educación y diversidad del alumnado, su formación psicopedagógica y didáctica (inicial y permanente) y su perfeccionamiento e innovación constantes.

Hay muchas cosas por hacer, y lo peor es que, tal vez, ni siquiera hemos llegado a una reflexión estratégica conjunta porque, en ocasiones, hemos centrado nuestras energías y nuestro discurso en el ámbito de las intervenciones individuales e inconexas, en perjuicio de respuestas expresamente comunitarias. A pesar de todo, no queremos perder la visión esperanzadora que nos anima y nos compromete más cada día. Y es que, parafraseando a Víctor Erice (director de cine), "en la realidad está todo, sólo hay que fijarse".

\section{Soporte documental}

\section{A) Legislativo}

Orden Ministerial de 4 de junio de 1987, por la que se convocan Proyectos de Orientación Educativa en Centros de Enseñanzas Medias (B.O.E. de 6 de junio). 
Ley Orgánica 1/1990, de 3 de octubre, de Ordenación General del Sistema Educativo (B.O.E. de 4 de octubre).

Real Decreto 1701/1991, de 29 de noviembre, por el que se establecen especialidades del Cuerpo de Profesores de Enseñanza Secundaria y se crea la especialidad de Psicología y Pedagogía (B.O.E. de 2 de marzo).

Orden de 27 de abril de 1992, por la que se dictan instrucciones para la implantación anticipada del Segundo Ciclo de Educación Secundaria Obligatoria y se especifican las tareas prioritarias que han de llevar a cabo los Departamentos de Orientación y la dedicación docente de sus miembros (B.O.E. de 8 de mayo).

Real Decreto 83/1996, de 26 de enero, por el que se aprueba el Reglamento Orgánico de los Institutos de Educación Secundaria (B.O.E. de 21 de febrero).

Resolución de 29 de abril de 1996, de la Dirección General de Centros Escolares, sobre organización de los Departamentos de Orientación en los Institutos de Educación Secundaria (B.O.E. de 31 de mayo).

Circular de 30 de abril de 1996, de la Dirección General de Renovación Pedagógica, por la que se regulan los aspectos referidos a la elaboración y desarrollo del Plan de Actividades de los Departamentos de Orientación (B.O.M.E.C. de 13 de mayo).

B) Bibliográfico

ÁLVAREZ, L. y SOLER, E. (2 ${ }^{\underline{a}}$ Ed. 1997). La diversidad en la práctica. Modelos de orientación y tutoría. Madrid: CCS.

ÁlVAREZ GONZÁLEZ, M. Y BISQUERRA ALZINA, R. Coords.(1996). Manual de orientación y tutoría. Barcelona: Praxis.

ÁLVAREZ ROJO, V. (1994). Orientación educativa y acción orientadora. Relaciones entre la teoría y la práctica. Madrid: EOS.

ARROYO, A., CASTELO, A. y PUEYO, Ma‥C. (1994): El Departamento de Orientación: Atención a la diversidad. Madrid: MEC/Narcea.

BORONAT, J. y CANO, R. (1998). "El Departamento de Orientación". En BISQUERRA, R. (Coord): Modelos de Orientación e Intervención Psicopedagógica. Barcelona: Praxis.

FOSSATI, R. y BENAVENT, A. (1996). "El Departamento de Orientación". En ALVAREZ, M. y BISQUERRA, R. (Coord): Manual de Orientación y Tutoría. Barcelona: Praxis.

GARCÍA REQUENA, F. (1997). Organización escolar de centros educativos. Málaga: Ediciones Aljibe.

HORTELANO, M.A. y JORGE, J. (1997): El Departamento de Orientación. Guía y documentos para el quehacer curricular y psicopedagógico. Salamanca: Amarú Ediciones.

MEC. (1992): Orientación y Tutoría en Secundaria. Madrid: Autor. 